\title{
Construcciones subjetivas ante el duelo de un cuerpo sano en adolescentes con diabetes mellitus 1
}

\section{Subjective constructions regarding mourning of a healthy body in adolescents with diabetes mellitus-1}

\author{
Laura Viviana Leal Guerrero ${ }^{I}$, Edith Gerardina Pompa Guajardo ${ }^{1}$ \\ y Martha Leticia Cabello Garza²
}

Citación: Leal Guerrero, L.V., Pompa Guajardo, E.G. y Cabello Garza, M.L. (2021). Construcciones subjetivas ante el duelo de un cuerpo sano en adolescentes con diabetes mellitus 1. Psicología y Salud, 31(1), 61-67. https://doi.org/10.25009/pys.v31i1.2676.

\section{RESUMEN}

\begin{abstract}
El propósito de esta investigación fue conocer una realidad social poco explorada desde el enfoque psicoanalítico: las construcciones subjetivas ante el duelo de un cuerpo sano en adolescentes con diabetes mellitus tipo 1. El estudio se llevó a cabo mediante un diseño metodológico de enfoque cualitativo, no experimental, transversal, exploratorio y descriptivo. Desde el paradigma psicoanalítico e interpretativo, está fundamentado en la fenomenología hermenéutica. Se realizaron entrevistas a profundidad a la población estudiada, constituida por cinco adolescentes con la referida enfermedad de entre 12 a 14 años de edad, adscritos a la Asociación Mexicana de Diabetes. Para la recolección de datos se utilizó la observación no participante en campamentos de verano, así como la entrevista a profundidad, el registro de opiniones y el análisis del discurso. Ser diagnosticado con diabetes en la adolescencia implica un doble duelo: la confrontación de las consecuencias orgánicas y los duelos propios de la adolescencia. El inconsciente social contribuye a estigmatizar ciertas enfermedades y, por consiguiente, a quienes las padecen, ocasionándoles dolor psíquico y desestabilidad en sus proyectos de vida. A través de la convivencia con la diabetes, los adolescentes perciben tener beneficios con la enfermedad, a la que adjetivan a través metáforas, manifestando una gran resistencia a renunciar a la misma.
\end{abstract}

Palabras clave: Adolescencia; Diabetes mellitus tipo 1; Investigación cualitativa; Duelo; Construcciones subjetivas.

\begin{abstract}
The purpose of the present study was to probe an unexplored social reality from a psychoanalytic approach. The main objective is to analyze subjective constructions associated to mourning of a healthy body in adolescents suffering from diabetes mellitus 1. The study's methodology involved a qualitative, non-experimental, transversal, exploratory and descriptive approach. Our psychoanalytic and interpretative paradigm was based on the hermeneutical phenomenology. In-depth interviews were conducted in participants which consisted of five adolescents with DM 1, between 12 and 14 years of age, registered in the Mexican Diabetes Association. Data were collected through non-participant observation during the association's summer camps, as well as in-depth interviews that registered opinions, and discourse analysis. Being diagnosed with diabetes in adolescence implies double mourning: coping with the organic consequences of the disease in addition to usual losses of adolescence. Social unconscious contributes to stigmatize certain diseases and, therefore,
\end{abstract}

\footnotetext{
${ }^{1}$ Facultad de Filosofía y Letras, Dr. Canseco 110, Col. Mitras Centro, Ciudad Universitaria, 64460 San Nicolás de los Garza, N.L., México, correos electrónicos: clinicapsicoanalitica1@outlook.com y edithpompag@gmail.com. Artículo recibido el 9 de noviembre de 2018 y aceptado el 23 de mayo de 2019.

${ }^{2}$ Academia Nacional de Investigación en Trabajo Social, Alondra 120, Col. Cuauhtémoc, 2o Sector, 66450 San Nicolás de los Garza, N.L., México, correo electrónico: marthacabello1@gmail.com (correspondencia).
} 
to those who suffer them, causing psychic pain and a destabilization of life projects. Through coexistence with diabetes, adolescents perceive benefits regarding the disease, they assign adjectives through metaphors while showing great resistance to renouncing to it.

Keywords: Adolescence; Diabetes mellitus type 1; Qualitative research; Mourning; Subjective constructions.

$\mathrm{H}$ ablar de la adolescencia es abordar una metamorfosis del sujeto en la que surgen cambios físicos propios de la pubertad, así como cambios psíquicos y emocionales ante los que se viven constantes pérdidas -en relación con el cuerpo- con los objetos internos y externos. La adolescencia es casi por definición un periodo de transición. El adolescente se encuentra en un proceso de cambio en el que vive el duelo por el cuerpo infantil perdido, por el rol y la identidad infantil y por los padres de la infancia (Fernández, 1981). Al buscar su identidad, el adolescente tiende a integrarse a grupos, expresa estados de ánimo inestables, fantasea, evoluciona sexualmente; en fin, sufre tan variados cambios que incluso sería anormal que mantuviera el equilibrio estable durante el proceso.

A lo antes apuntado podría considerársele una adolescencia "normal". Mas, ¿qué sucede cuando a esa transición de cambios se suma una enfermedad crónico degenerativa como la diabetes? Una vez diagnosticados, los adolescentes requieren tener un control estricto de su dieta alimenticia, un alto nivel de autocontrol y disciplina, cuidados diversos que implican un cambio radical en sus vidas al convertirse en insulinodependientes y sufrir por ello diversas restricciones. En este caso ya no se está hablando de cualquier adolescente, sino de un adolescente enfermo. Cualquier enfermedad crónico degenerativa implica que las personas que las padecen se vean obligadas a emprender drásticas transformaciones en su vida, en la que se manifiesta no solamente un malestar físico, sino además un malestar psíquico que envuelve tanto al enfermo como a su familia.

Se puede precisar el problema que se suscita mediante cifras. La Federación Internacional de Diabetes (FID) (2015) estima que aproximadamente 425 millones de personas en el mundo su- fren la enfermedad, y que el número de enfermos aumentará en 2040 a más de 642 millones. Las estimaciones de la diabetes tipo 1 en adolescentes y jóvenes también muestra aumentos inexplicables en varias regiones, y se calcula que actualmente hay más de medio millón de menores con este padecimiento (FID, 2015).

En el continente americano hay aproximadamente 62.8 millones de personas con diabetes. México ocupa el quinto lugar mundial de personas en esta condición, esto es, doce millones (FID, 2015). Según la Encuesta Nacional de Salud y Nutrición (ENSANUT 2016) (Instituto Nacional de Salud Pública (INSP), 2012, 2016), 0.68\% de adolescentes ya ha sido diagnosticado con diabetes, lo que representa alrededor de 155 mil individuos en este rango de edad. En el estado de Nuevo León la cifra es de 698,018, equivalente a 14.6\%; es decir, tres de cada diez neoleoneses padecen esta enfermedad (INSP, 2016).

La diabetes mellitus tipo 1 (DM 1 en lo sucesivo) es causada por una reacción autoinmune, en la que el sistema de defensa del cuerpo ataca las células beta productoras de insulina en el páncreas. Como resultado, el cuerpo ya no puede producir la que necesita. La enfermedad puede afectar a personas de cualquier edad, pero su aparición ocurre normalmente en niños y jóvenes adultos. Las personas con este tipo de diabetes necesitan insulina diariamente para controlar sus niveles de glucosa en la sangre.

La DM 1 en los adolescentes muestra un panorama crítico debido a que atraviesan una serie de cambios propios de la etapa, a los que se añaden los propios de la enfermedad, de modo que a partir del diagnóstico sobrevienen una serie de cambios adicionales ante los cuales los adolescentes deben adoptar y asumir diferentes actitudes que dependerán de su historia personal, de su contexto familiar y social y de la forma como se ha presentado el diagnóstico. Esta situación puede ser o no disruptiva, lo que dependerá de las construcciones subjetivas que el adolescente elabore ante la pérdida de un cuerpo sano.

Desde una perspectiva psicoanalítica, la DM 1 tiene importantes efectos psíquicos ya que genera en los adolescentes -sobre todo en relación con la representación del cuerpo y los procesos de duelo- una aflicción por el sentimiento de la pérdida 
de la salud. Es entonces cuando los adolescentes se encuentran en ese doble duelo: el propio de la adolescencia y el de la pérdida del cuerpo sano. El psicoanálisis concibe al sujeto como dividido, inconsciente, atravesado por el lenguaje; dicha concepción permite definir a partir de allí un cuerpo, que será singular para cada individuo.

En este sentido, el psicoanálisis habla sobre una significación simbólica del cuerpo, de la imagen corporal y del órgano que se enferma. Si bien Freud (1917) teorizó desde el plano psíquico, da a entender en su teoría que el aparato psíquico no es algo desprendido del cuerpo, sino que, por el contrario, tiene su anclaje en lo biológico.

En este proceso de enfermar, el cuerpo biológico, quien detenta la afección, es un espacio íntimo de experiencias donde las enfermedades se instalan y donde los cambios corporales que se suscitan a lo largo de este proceso suelen provocar reacciones emocionales de pérdida en las personas: pérdida del cuerpo infantil en la adolescencia, pérdida de la capacidad reproductora en la menopausia, pérdida "de la propia impermanencia", al decir de Alizade (2000).

La enfermedad crónica no es un entorno suficientemente bueno para desarrollar convenientemente una adolescencia (Lachal, 2002). Malka, Togora, Chocard, Faure y Duverger (2007) afirman que la enfermedad crónica trastorna las relaciones, y que los efectos de la enfermedad inciden en el narcisismo del adolescente. Su cuerpo, en pleno proceso de sexuación, es sitiado por la enfermedad crónica. Desde esta perspectiva, se asume que, identificando los conflictos psíquicos por los que atraviesa el adolescente enfermo de diabetes, se puede generar conocimiento, primeramente para conocer por medio de su narrativa lo que está viviendo $\mathrm{y}$, con base en ello, crear una propuesta de intervención psicológica que dote al paciente de las herramientas necesarias para el control adecuado de su enfermedad.

Si bien existen varias teorías relacionadas con el duelo, la de Kübler-Ross (1969) señala que la negación, la rabia/ira, la negociación, la depresión y la aceptación son etapas que ayudan a comprender el proceso que atraviesa un enfermo terminal al vislumbrar su propia muerte o, mejor dicho, la pérdida de la propia vida. Hay una misteriosa presencia de la subjetividad en los enfermos crónicos, presencia que determina un no saber cómo responder a la queja permanente que los caracteriza al no saber qué hacer con ellos mismos (Gallo, 2002).

De acuerdo a Foucault (2000), la enfermedad no solo implica una situación reconocida por el propio enfermo como observador objetivo y por el médico como científico, sino que además plantea la necesidad de enfocar el enorme espectro de significados que circulan entre las personas que rodean al enfermo. La cuestión está en ver cómo se construye la concepción de la desviación en ese entorno y qué consecuencias tiene. Al reconocer la enfermedad en una persona, las demás moldean sus conductas frente a ella, y con esto modifican su propia subjetividad. La enfermedad cobra así una materialidad distinta a la del simple mal, convirtiéndose en un estado social. Como tal, dicho estado varía con la cultura y con los significados que se le atribuyan.

Por otra parte, el enfermo crónico parece enamorado de su mal; a pesar de quejarse, no se encuentra dispuesto a abandonar su enfermedad porque esta se ha convertido en su "compañera inseparable", como un elemento que lo representa ante el otro. Quien posee una enfermedad crónica está tan bien educado acerca de su enfermedad que el problema no es orientarle para que el tratamiento tenga éxito, sino cómo descentrar su ser de la identificación con el mal (Gallo, 2002).

De acuerdo a lo anterior, el objetivo del presente trabajo fue conocer las construcciones subjetivas ante el duelo de un cuerpo sano en adolescentes con DM 1 mediante un diseño de investigación cualitativo y desde una perspectiva fenomenológico-hermenéutica.

\section{MÉTODO}

Tal diseño se eligió en función del objeto de estudio, que son los adolescentes; el nivel de comprensión, en el enfoque cualitativo, se alcanzó sobre la representación subjetiva que tiene el adolescente de su padecimiento. Este trabajo da cuenta de un estudio no experimental, exploratorio y descriptivo, que a través de un diseño narrativo-interpretativo busca conocer esa realidad social tan escasamente investigada por el psicoanálisis, con 
el propósito principal de analizar las construcciones subjetivas ante el duelo de un cuerpo sano en adolescentes con DM 1.

\section{Participantes}

Se empleó una muestra por conveniencia compuesta por adolescentes diagnosticados con DM 1 que cumplieron con los siguientes criterios de inclusión: tener entre 12 y 14 años de edad, estar incorporados a la Asociación Mexicana de Diabetes, y ser pacientes con un diagnóstico no mayor a dos años. Se excluyeron los adolescentes con DM 2 o con diabetes gestacional. La muestra final estuvo constituida por cinco adolescentes: tres mujeres y dos hombres.

\section{Instrumentos}

Las técnicas de recolección de datos fueron las entrevistas a profundidad, la observación no participante y el registro de opiniones durante una estadía en un campamento de verano.

\section{Procedimiento}

Antes de llevar a cabo el trabajo de recolección de información, se les leyó la carta de consentimiento en que se informaba a los padres de los adolescentes los objetivos de la investigación, la colaboración que se esperaba de ellos y el asentimiento informado de los participantes. Se les pidió asimismo su autorización para grabar las entrevistas, así como expresar su consentimiento para utilizar los resultados con fines de investigación.

Durante la entrevista a profundidad, en la que el entrevistado se explaya libremente sobre sus sentimientos y pensamientos sin un orden predeterminado para tratar los temas, se sigue el discurso del entrevistado con preguntas pertinentes que no están previamente estructuradas sino retomadas del propio discurso del sujeto, lo que lo lleva a profundizar en él. Las entrevistas fueron grabadas y transcritas fielmente, y se utilizó el software Atlas ti 7 para el análisis de los datos. Se inició el trabajo de análisis seleccionando segmentos de la entrevista y creando las citas y las codificaciones del material significativo. La mayoría de los códigos fueron emergiendo conforme se avanzaba en el análisis de las entrevistas. Después se hicieron las familias de códigos o categorías. Una vez terminada la codificación y tras leer repetidamente las entrevistas, se identificaron los elementos repetitivos que hicieran possible construir las categorías de análisis, que consistieron en la construcción subjetiva y en sus subcategorías (estigma social, prejuicios ante la enfermedad, representación subjetiva ante la enfermedad y proyecto de vida). Las entrevistas a profundidad se efectuaron de forma individual en los cubículos de posgrado de la Facultad de Psicología de la Universidad Autónoma de Nuevo León.

\section{Guía de entrevista}

La entrevista contiene preguntas como las siguientes, las que, como se dice antes, atienden lo que expresa libremente el participante: "Cuéntame un poco sobre ese momento en que te dijeron que tenías diabetes; ¿ y luego qué pasó?; ¿te has puesto a pensar por qué enfermaste?; cuéntame cómo era tu vida hace dos años; ¿ha cambiado tu forma de pensar, sentir o vivir desde el momento en que te diagnosticaron la enfermedad?; cuéntame cómo es para ti tener una enfermedad como la diabetes; ¿cómo representas la diabetes en tu vida?; ¿cambió el trato que te daban los demás?; ¿cómo piensas que te perciben los otros?; ¿cambiaron tus planes a futuro después de que te diagnosticaron la diabetes?; ¿hay alguna otra cosa que quieras agregar que te parezca importante sobre todo lo que hemos platicado?”.

Al concluir la entrevista se integra la interpretación cualitativa de la construcción subjetiva de los adolescentes y se triangulan los datos con la observación no participante y los registros de opinión. La observación no participante es otra de las técnicas utilizadas en esta investigación, la cual participa en los procedimientos para obtener información del objeto de estudio empleando los sentidos con determinada lógica relacional de los hechos, y como instrumento de investigación utilizado sistemáticamente para obtener información a través de los principios del método científico al buscar la validez y confiabilidad de los datos obtenidos.

Se efectuaron las observaciones en los campamentos de verano de Teotepec, ubicados en la 
sierra de Arteaga (Coahuila), y organizados por la Asociación Mexicana de Diabetes. Durante siete días días se hizo una observación no participante, directa y colectiva a 67 adolescentes, así como a médicos residentes de endocrinología, quienes estaban a cargo de los monitoreos, el control de las dosis de insulina y el conteo de carbohidratos, así como otros especialistas en diabetes.

La última técnica para la recolección de datos fue el registro de opinión que los especialistas y los propios adolescentes hacían en diversos momentos de su estancia en el campamento, con el objetivo de incluir las vivencias de los propios actores enmarcadas en las pláticas informales. Por ultimo, se hizo la triangulación de la observación no participante, los registros de opinión y las entrevistas en profundidad.

\section{RESULTADOS}

La categoría que se analizó fue la llamada "construcción subjetiva". En esta se muestra la manera en que los adolescentes viven y piensan la enfermedad, la cual se dividió en siete códigos: Construcción subjetiva, ¿Por qué a mí?, Estigma social por tener un cuerpo enfermo, Metáforas de la enfermedad, Beneficios de estar enfermo, Proyecto de vida y Renunciar a la enfermedad. Dicha categoría provino de los objetivos específicos de la investigación que, como ya se dijo, converge en determinadas subcategorías de análisis.

Lo anterior se muestra en el siguiente diagrama:

Figura 1. Representación gráfica de la categoría construcción subjetiva.

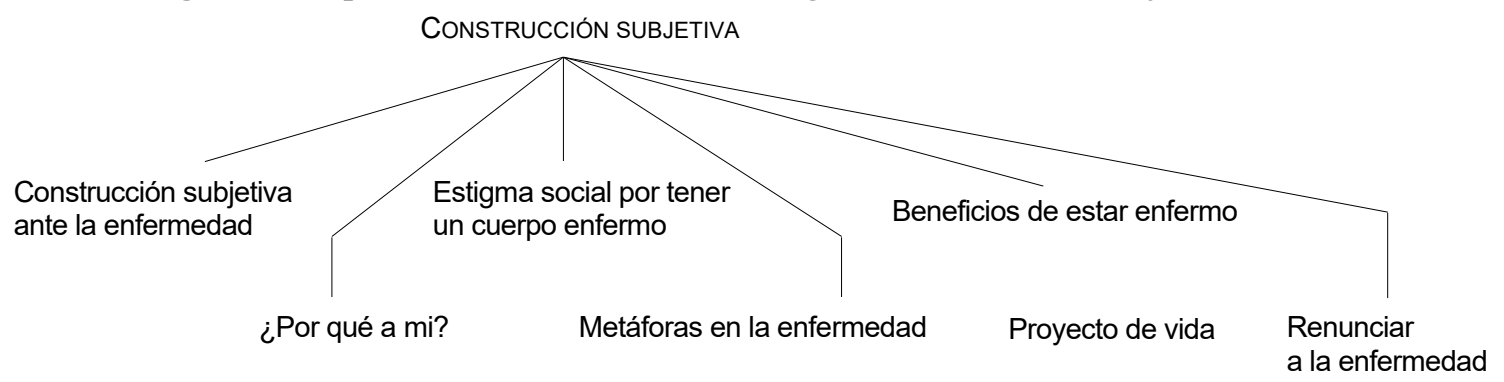

El diagnóstico de diabetes implicó para los adolescentes la confrontación de las consecuencias orgánicas y psicológicas propias de una enfermedad llena de significaciones subjetivas; cada adolescente había vivido de maneras diferentes este proceso, según lo atravesaba su subjetividad al concebirse como enfermo.

Los adolescentes manifestaban la primera etapa del duelo: la negación, desde el momento en que supieron que padecían una enfermedad crónica y permanente (Suris, Michaud y Viner, 2004)). Fue entonces que habían empezado a experimentar dolor psíquico por la concientización de las pérdidas ya sufridas y las que faltaban.

En ese sentido es posible hablar de un doble duelo, pues se valora más la salud cuando ya se ha perdido. Experimentaban el malestar físico y la disrupción psíquica que surge al ver los cambios entre su estilo de vida anterior (carente de prohibiciones) y los que debían llevar en el futuro. Se percibían de un modo diferente, pues la diabetes implica una alimentación restrictiva y una rutina de chequeo que no hacían sus pares. Al transcurrir el tiempo los adolescentes diabéticos constatan que la enfermedad es para siempre y una parte esencial de sus vidas.

Hay otro doble duelo, manifestado en la rebeldía con la cual responden a sus temores y ansiedades por no saber lo que le ocurre a su cuerpo desde dos vertientes: la propia de su desarrollo y la de la enfermedad.

No obstante, a través de la convivencia con la diabetes los adolescentes perciben tener ciertos beneficios y ganancias debidos a la enfermedad, al grado de expresar que "no se arrepienten de tener diabetes", como la unión familiar resultante del diagnóstico, la pérdida de peso y la mejora de sus hábitos alimenticios y de su condición física.

La diabetes impacta en el narcisismo propio de la adolescencia, ese sentimiento de "indefensión" desde la mirada de un Otro lleno de estigmas que desencadenan prejuicios. En el inconsciente social existe un entramado complejo de significaciones y patrones previos que operan en un nivel 
preconsciente; en ese proceso, los otros contribuyen a estigmatizar enfermedades como la diabetes $\mathrm{y}$, por ende, a quien la padece, lo que aumenta sus sentimientos de inferioridad y vulnerabilidad.

La imagen del cuerpo es eminentemente inconsciente; sin embargo, cuando se asocia al lenguaje consciente, es cuando se utilizan metáforas y metonimias. En este estudio se apreció que los adolescentes utilizaban metáforas referidas a su enfermedad como respuesta a la estigmatización por parte de los demás, incluyendo familiares.

Finalmente, en relación al proyecto de vida, dado que los participantes se hallaban en esta etapa y estaban apenas formando su identidad y preguntándose quiénes eran y a qué aspiraban, sus discursos iban principalmente dirigidos al deseo surgido tras la enfermedad. La mayoría de los entrevistados tenían planeado ser psicólogos como primera opción, sin descartar la posibilidad de estudiar nutrición; cabe mencionar que el interés por dichas profesiones apareció después del diagnóstico de diabetes. En este sentido, se hallaron elementos muy similares, como terminar sus estudios y ejercer una profesión para ayudar a otros que viven la misma situación. El proyecto de vida implica para ellos un rompimiento de expectativas; al ser la enfermedad un obstáculo, genera sentimientos de tristeza, frustración, angustia y miedo; contrariamente a lo que cabría esperar. Todos los adolescentes entrevistados manifestaron una gran resistencia, consciente o inconsciente, a renunciar a su enfermedad porque esta les generaba ciertas ganancias, pues el síntoma le da un asiento a la angustia, así como un nombre, y puede subsanar lo indeterminado que dicho malestar genera, quedando velada por la enfermedad que le da un nombre al sujeto. Es como un síntoma, pero real. En este caso, el padecimiento también funciona así, como un síntoma que le da un nombre al sujeto. Si bien al enfermar de diabetes los adolescentes se encuentran en una forma de pérdida, el ubicar lo perdido les permite salir del riesgo del dolor inconsciente; es decir, ahora saben lo que perdieron.

\section{DISCUSIÓN}

El sujeto que enferma produce cambios corporales, tiene la necesidad de transitar por diversos duelos, por las distintas pérdidas que ocasionan los tratamientos, por ese cuerpo dañado, por sus ideales narcisistas y por un modo de vida que ya no será igual al de los demás adolescentes.

El duelo, como proceso de trabajo y elaboración psíquico, permite la reconstrucción afectiva y la resignificación de la vida ante la enfermedad.

Las rutinas y cuidados que la diabetes mellitus requiere ponen en juego la relación familiar al crear conflictos entre padres e hijos; pues estos últimos se dicen hartos de la presión y la sobreprotección de los primeros.

Algunos de los participantes en el estudio tendían a no seguir las indicaciones en cuanto al consumo de alimentos cuando convivían con otros adolescentes. Esta pulsión de muerte se propiciaba cuando mostraban enojo contra sus padres, como una forma de castigo hacia ellos.

Durante los tratamientos deben enfrentarse a las repercusiones psíquicas y sociales de la enfermedad, y por tanto a la experiencia de pérdida ya descrita anteriormente. En la experiencia del tiempo subjetivo la persona hace una parada psíquica y se percata de que hay un antes y un después. Tendrá que hacerse cargo de los cambios desencadenados por la diabetes y adquirir una responsabilidad para asumir el autocontrol y una "responsabilidad de sí".

Los adolescentes, frente al desconcierto de lo irremediable, de los procesos de un doble duelo, de los estigmas sociales que viven día a día, necesitan hablar y ser escuchados; necesitan poner en palabras su dolor, en las que es frecuente la utilización de la metáfora para intentar apropiarse de ese sentir donde reina el dolor y el goce, y donde al enfermo le faltan las palabras.

Es por ello que se considera fundamental abrir el espacio a la palabra del adolescente con un cuerpo enfermo y hacer un acompañamiento como clínica de la escucha de la palabra, mediante la cual, en cuanto sujeto, pueda recuperar su lugar y construir un saber sobre lo acontecido a su cuerpo. Esta práctica clínica está en estrecha relación con el psicoanálisis y procura abrir espacios al síntoma como fuente de saber.

La diabetes golpea al narcisismo propio de la adolescencia, ese sentimiento de indefensión desde la mirada de un Otro pleno de estigmas que desembocan en prejuicios. En el inconsciente so- 
cial existe un entramado complejo de significaciones y patrones previos que operan en un nivel preconsciente en ese proceso en el que los otros contribuyen a estigmatizar ciertas enfermedades y a quienes las padecen, lo que acrecenta los sentimientos de inferioridad y vulnerabilidad.

Por otro lado, los adolescentes en estudio se identifican con la diabetes, es decir, se apropian de la enfermedad, lo que implica un acomodamiento del yo a la enfermedad que los conduce a descubrir que, mediante la diabetes, pueden llegar a obtener ciertas gratificaciones: al tener un cuerpo enfermo, ya no deben hacer ningún esfuerzo para ser reconocidos y recibir aprecio o amor.

Según lo antes dicho, los adolescentes se resisten a "soltar" la enfermedad; a pesar de sus quejas, no están dispuestos a renunciar a ella, que es el elemento básico que lo representa para un otro. En este sentido, la enfermedad ya no es vivida como un cuerpo extraño que debe extirparse del organismo y del ser, sino como una forma de representación en la que el sujeto no está dispuesto a curarse.
Uno de los resultados más significativos, aunque poco esperados del estudio, es que en la totalidad de los casos manifestaron no querer curarse, lo que se interpreta como que dejar de estar enfermos significa perder todo aquello que lograron obtener en poco tiempo; quizá lo que no consiguieron del Otro en tantos años de conflicto psíquico, lograron a través de la diabetes que ese Otro atendiera sus demandas: ahora tienen un lugar y son reconocidos por ello. El malestar físico los libra del malestar psicológico, y así logran hacer ese llamado al Otro y finalmente ser atendidos, de manera que lo que no fue en un momento tramitado en palabras se convierte en actos que se repiten constantemente. No desear desprenderse de ese beneficio representa la pérdida real del objeto amado, simbolizado en la respuesta recibida por el otro social.

Finalmente, si bien este estudio no permite establecer generalizaciones de los resultados obtenidos, es una aproximación válida para intentar comprender "algo más" de lo que ocurre con esta enfermedad.

\section{REFERENCIAS}

Alizade, A.M. (1999). Duelos del cuerpo. Memorias del Primer Congreso de Psicoanálisis y XI Jornadas Científicas “Los duelos y sus destinos. Depresiones hoy”. Asunción (Paraguay): Asociación Psicoanalítica de Paraguay.

Doltó, F. (1991). La causa de los adolescentes. Barcelona: Seix Barral.

Federación Internacional de Diabetes (2015). Atlas de la diabetes de la FID (7a ed.). Bruselas: FID. Recuperado en http://www. idf.org/diabetesatlas.

Fernández, O. (1981). El trabajo de duelo durante la adolescencia. En S. Quiroga (Comp.): Adolescencia: de la metapsicología a la clínica. Buenos Aires: Amorrotu.

Foucault, M. (1983). Las palabras y las cosas. México: Siglo XXI.

Freud, S. (1917). Duelo y melancolía. En S. Freud: Obras completas, vol. XIV. Buenos Aires: Amorrortu.

Gallo, H. (2002). Cuerpo y enfermedad: Una aproximación psicoanalítica. Desde el Jardín de Freud, 2, 120-127.

Instituto Nacional de Salud Pública (2012). Encuesta Nacional de Salud y Nutrición 2012 (ENSANUT 2012). Cuernavaca (México): INSP.

Instituto Nacional de Salud Pública (2016). Encuesta Nacional de Salud y Nutrición (ENSANUT 2016). Informe final de resultados. Cuernavaca (México): INSP.

Kübler-Ross, E. (1969). On death and dying. New York: Scribner.

Lachal, C. (2002). La construcción de la subjetividad y el enlace a la adolescencia. Campo Psicosomático, 25(1), 25-47.

Malka, J., Togora, A., Chocard A.-S., Faure, K. y Duverger, P. (2007). Réflexion sur l'impact psychique de la maladie somatique chronique chez l'adolescent. Neuropsychiatrie de l'Enfant et de l'Adolescence, 55(3), 149-153.

Suris, J.C., Michaud, P.A. y Viner, R. (2004). The adolescent with chronic condition. Part I: Developmental issues. Archives of Disease in Childhood: Education and Practic, 89, 938-942. 